\title{
Modelling the Influence of Social Media on Collective Opinion
}

\author{
Teruaki Ohnishi \\ Urayasu Branch, Institute of Science and Technology for Society, Urayasu, Chiba, Japan
}

Email address:

ohnishiteruaki@yahoo.co.jp

\section{To cite this article:}

Teruaki Ohnishi. Modelling the Influence of Social Media on Collective Opinion. American Journal of Physics and Applications. Vol. 8, No. 6, 2020, pp. 78-87. doi: 10.11648/j.ajpa.20200806.11

Received: October 22, 2020; Accepted: November 13, 2020; Published: November 24, 2020

\begin{abstract}
When we divide our present society into real and cyber worlds, there exist no clear data on how the public attitude or opinion is formed and on what sort of opinion distribution is realized in the cyber world. We propose a methodology for the model calculation with which we can compare the observation of the public opinion formed under the environment of social media in the cyber world. The public viewpoint or the opinion about a certain matter, together with the standpoint of the information provided by the social media, can not be given by some discrete values, but they make fuzzy distributions within certain ranges of opinion around certain central values. With the assumption that the variation of the public opinion originates from the emotional contagion induced by the contact of the public with the social media, and that the force realized by this contagion is given in terms of the common area of such fuzzy distributions of the public opinion and the information on the social media, we derived an equation of motion for the variation of public opinion. By further assuming that the information diffuses from a top toward a bottom of a ramified tree structure of node networks, we exemplified some characteristic patterns of the distribution of collective opinion including the effect of echo-chamber, which are realized under certain input spectra of the information on the social media. Moreover, by using the observed data for the 2016 USA President election as an input, we made clear that the reversal of the approval rating might possibly occur between the political right and left wings in so far as the response character of supporters to the social media differ depending on the political situation of the public.
\end{abstract}

Keywords: Collective Public Opinion, Social Media, Information Diffusion, Emotional Contagion, Fuzzy Function, Equation of Motion of Public Opinion, 2016 USA President Election

\section{Introduction}

Today we are living in an environment full of information of various matters. Every person as a member of collective society forms and changes opinions of his own through the direct conversation and discussion with the surrounding member. Opinion dynamics is a methodology to describe such a process by physical mathematics. When the sufficient exchange of opinions is realized in a society, several collective opinions are formed and the opinion of each member is finally absorbed by one of such collective opinions. A representative model of such dynamics is the bounded confidence model $\mathrm{BCM}$ ) [1] and its renewal versions [2]. With regard to the variation of individual attitude and public opinion, the susceptible-infected-recovered (SIR) model and the immune model are also proposed [3-5]. In these models the individual attitude or opinion are treated as discrete values so that a tacit assumption is made as there exist no vague portions in our mind. Such a discussion, however, is limited to our real world.

The information environment surrounding our lives is in a form of a superposed state of the real world originating from newspapers, the television and direct human relations, and the cyber world from the Internet and SNS so that the public opinion or the recognition are formed under the integrated influence of these two worlds [6]. Here the cyber world means the virtual space of which a main function is the information exchange through the SNS such as Twitter, Facebook, YouTube etc. and does not necessarily mean the site that gives news or other general information in a unidirectional way to the society. The way to transfer information and the relation between the relevant people in the cyber world are clearly different from the real world. The opinion dynamics and the rumor dynamics for the cyber world prevailing at present are mostly of the extension of 
those in the real world [3-5, 7-11]. The investigation of models specific to the cyber world is, therefore, required at present where consideration is made about the input way of information in the cyber network along with the way of its diffusion in the network. [12, 13]. Moreover it is also required to consider, from a different point of view from the past, how the public recognition is influenced and changed by the social media in that world. With regard to these problems we pay attention to the following two points.

The first is the non-discreteness of the public cognition so that it is not appropriate to describe it by a single value. The individual attitude and opinion can not usually be given by a definite value or a value within a narrow range even in the case without any remarkable perturbation from the outside. The attitude and opinion are varied under the influence of various matters and concerns surrounding ourselves [14]. Therefore to quantify the individual attitude and opinion, they must be treated as if they fuzzily distribute around some central values. The same situation is held in considering the quality of information, which is not objectively constant but varies anyway depending on the susceptibility of the individual people. Hence in quantifying the quality of information in the modeling, it also must be treated as if its value distributes fuzzily.

The second point to be noticed is that the definitive method such as traditional BCM model can not be applied when the fuzzy environment of information exerts on the fuzzy state of public opinion. The public attitude and opinion will be varied under the influence of emotional contagion of the information environment that is vague and fuzzy [15-20]. Hence the fuzzy theory $[21,22]$ may possibly be introduced in treating the opinion dynamics.

In this paper we consider the society in the cyber world in the form of ramified stratum with information diffusing from the upper layer toward the bottom one, where assumed is the network of nodes, instead of the public, in a small world type [23-25]. Although the nodes are assumed to receive the information common to all nodes and to access to common sites of information, independently to each other, no emotional exchange which influences the change of opinion is assumed to occur within the member of the network.

In the next Sec. II with such prerequisites, we study a model of opinion change in cyber world. The attitude or the opinion in this model is of the cyber world for the public with strong relation to the cyber world in everyday life, so that they do not correspond to the general averages throughout the society including the real world. In Sec. III we show some calculations together with the figures indicating echo-chamber effect [26, 27], and in Sec. IV, study is made on the 2016 USA President election, showing the time variation of the number of political supporters on the right and left wings [28-30]. Concluding remarks are given in Sec. V.

\section{Model}

The actual public opinion in our day is not given until we take into consideration of both contributions from the real and the cyber worlds. The following model is only for the effect from the cyber world. We study on what extent of the collective public opinion is influenced when some information on a certain matter is repeatedly distributed through cyber networks.

The information distributed in the society regarding a certain matter, together with the public opinion of the matter, have each value of their standpoints. We will call such value as an opinion hereafter, giving such an opinion with a numeral $x \in[0,1]$. The transmission of information or its diffusion in the cyber world is exerted through cyber networks, where the information is first released from the server (the information sender) to the top node $\mathrm{m}_{1}$ (the initial spreader) of each network. The $m_{1}$ then transfers the information to the next node $\mathrm{m}_{2 \mathrm{i}}\left(\mathrm{i}=1 \ldots \mathrm{im}_{2}\right)$ on the second layer, the node $\mathrm{m}_{2 \mathrm{i}}$ further to the $\mathrm{m}_{3 \mathrm{i}}\left(\mathrm{i}=1 \ldots \mathrm{im}_{3}\right)$ on the third layer, and so on. Here the node corresponds to each people, and $\mathrm{n}\left(\mathrm{m}_{\mathrm{fi}}\right)$ branches or edges spread from one node $\mathrm{m}_{\ell \mathrm{i}}$ on an upper layer to connect to the nodes on the lower layer. This is a simple image of information transfer of our case. In the case of such a ramified structure, the number of nodes on a layer grows with increasing the layer number (with becoming deep into the network) in so far as the condition $n\left(m_{\ell i}\right)>1$ holds. Assuming the existence of only one server for simplicity, we show our model as shown in Figure 1.
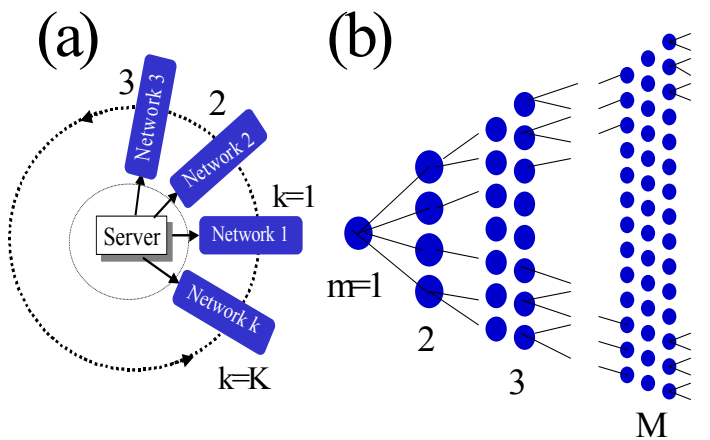

Figure 1. Schematic figure for our cyber network structure. (a) K networks connected to a server. (b) M layers in one network.

\subsection{Cyber Network}

The number distribution of nodes in the network is assumed as [23-25]

$$
\varphi_{k}\left(n_{k}\right) d n_{k}=\alpha_{k} \cdot n_{k}^{-\beta} d n_{k}
$$

where $k$ represents the $k^{\prime}$ th network connected to the server, $\alpha_{k}$ is a constant, and $n_{k}$ is the number of ramification of the node on an arbitrary layer in the network $k$. Although we set $\beta=1.5$ in what follows, our qualitative results do not depend on its value. The server is assumed to release, at the same time, the information or the message common to $K$ networks with different structures. Figure 2(a) shows the number of nodes up to the $5^{\prime}$ th layer $(M=5$ in Figure $1(\mathrm{~b}))$ averaged over 800 networks $(K=800)$ that are produced by using Eq.(1). The average number of nodes belonging to the $m^{\prime}$ th layer $<N_{m}>$ is given by $<N_{m}>=m^{3.518}$ with $\beta=1.5$. On the other hand, Figure 2(b) shows the frequency of the total number of nodes up to 
the 5 'th layer for our 800 networks. So far as we discuss the frequency up to the 5 'th layer, it is $\sim 22 \%$ where the total number of nodes does not exceed 50 , and $\sim 12 \%$ for both the total numbers with $50 \sim 100$ and 100 150.
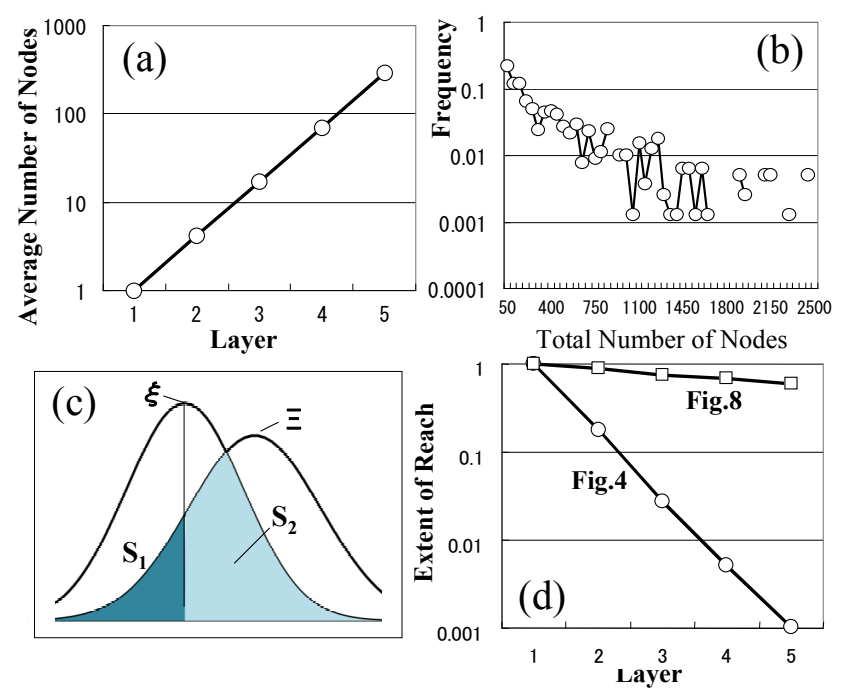

Figure 2. (a) Average number of nodes belonging to each layer up to the 5 'th layer. (b) Frequency distribution of the total number of nodes $N_{k}$ belonging to a single network. (c) Superimposed regions to be integrated. (d) The reach $\Omega_{m}(t)$, that is the degree of the transmission of information as a function of layer number up to which the information reaches, shown for two cases of Figure $4(t=0.1)$ and Figure $8(t=199)$ as for examples.

The information is assumed to release from the server at an arbitrary interval, and the node on a layer is also assumed to transfer (re-tweet) the received information to the node on the next layer with a probability $\omega$ which will be given later. Even if the information includes any references or links to the Internet or SNS sites, each node is assumed to receive the same and common contents as the top one. The time for the information to diffuse into the network is neglected for simplicity, assuming it to propagate without any delay of time. The collective opinion of the public in the cyber world is derived as the opinion averaged over all nodes belonging to $K$ networks.

The information released on a certain matter is assumed to have a view of value or the opinion $x$ of the matter with $x \in[0,1]$, which is called the opinion (of information) as before. Since each people or each node has its own subjectivity, the meaning of the information differs with each node so that the opinion of the information $x$ can not be discrete but probably distribute within a certain range of value around a center $\Xi$. Hence the stance, that is the opinion of the information received at a time $t$ by the node must have a sort of spectrum $\Psi(x, t: \Xi)$ with regard to $x$ and a central value $\Xi$.

$$
\Psi(x, t: \Xi) \equiv F_{s}(x, t: \Xi)
$$

where $F_{s}(x, t: \Xi)$ is the probability such that the information with the central opinion $\Xi$ is received as the one with the opinion $x$, and it is a function to give an extent of fuzziness of the information. The form of the function will be given later.

\subsection{Distribution of the Opinion of Nodes}

Each node shown in Figure 1(b) has the opinion in the range $[0,1]$. As already described the opinion of an individual node is not possibly a discrete value $[31,32]$ but vaguely distributes around a central value $\xi$ in actuality. This is because the public cognition or the opinion generally fluctuates in a certain range of value under the condition of varying social environment by the so-called opinion copulas or the topic contagion [14, 31, 32]. We assume the distribution function of the opinion of the node at a time $t, \Phi(x, t: \xi)$, as

$$
\Phi(x, t: \xi)=F_{n}(x, t: \xi) / \mathrm{G}
$$

where $F_{n}(x, t: \xi)$ is a function of $x$ fuzzily distributing around $\xi$. Such a function together with $F_{s}(x, t: \Xi)$ correspond to the so-called member function or the fuzzy function in the field of fuzzy theory $[21,22]$. The quantity $G$ is a normalization constant which makes the weight of each node as 1.0 in the range of $x \in[0,1]$. We set the form of those fuzzy functions as a type of normal distribution. Even when we adopt other forms of fuzzy function, our qualitative results are not essentially changed.

$$
F_{f}(x, t: X)=\frac{1}{\sqrt{2 \pi} \sigma_{f}} \exp \left\{-\frac{(x-X)^{2}}{2 \sigma_{f}^{2}}\right\}
$$

where $f=s$ or $n, X=\Xi$ or $\xi$, and $\sigma_{f}$ is a deviation giving the extent of dispersion. When we each is asked about the opinion of ourselves, the above equation with $f=n$ gives a probability to answer that it is $x$. Adopting the above Eq.(4), we give the $G$ in Eq.(3) as

$$
G=1-\pi^{-1 / 2} \cdot\left\{\operatorname{erfc}\left(\frac{1-\xi}{\sqrt{2} \sigma_{n}}\right)+\operatorname{erfc}\left(\frac{\xi}{\sqrt{2} \sigma_{n}}\right)\right\}
$$

where $\operatorname{erf} c(x)$ is Gauss' error function.

Since the opinion of an individual people in the real world has been assumed to change, for instance, through the interaction with the neighboring people, the extent of its change is derived depending on the number of surrounding people and the difference of opinions between people. When there exist emotional or sentimental factors between people, or linguistic and visual contact in the interpersonal interaction, the emotional contagion arises between the relevant people $[15,16,18,20,31,33]$. According to these viewpoints, the opinion change of our case also must be owed to the emotional reaction of nodes to the social media, that is, the emotional contagion, together with the rational reaction to the logical contents of the information. Since the opinions of the information and the node distribute around central values $\Xi$ and $\xi$, respectively, the extent of reaction of a node to the information may be given by a certain function of the following integration, that is the area $S$ of the superimposed part of those functions. 


$$
S(t: \Xi, \xi)=\int_{0}^{1} \Psi(x, t: \Xi) \otimes \Phi(x, t: \xi) d x
$$

where $\otimes$ represents the superimposed part of two functions. With our fuzzy functions given by Eq.(4), the $S(t: \Xi, \xi)$ is generally given by the sum of error function. Since the area of the function for each node is normalized as 1.0 (as Eq.(3)), the area given by Eq.(6) becomes to be an indicator to give a relative extent of influence of the node from the information.

We divide the superimposed part shown in Figure 2(c) into two parts $S_{1}$ and $\mathrm{S}_{2}$ on the left and right respective sides of the center $x=\xi$. In this case the $S_{2}$ can be interpreted as the contribution from the information with the center $\Xi$ to draw the node with the center $\xi$ toward the direction of the information itself, whereas the $S_{l}$ is the contribution from the information to move the node away from it. This is because the component $S_{2}$ situates on the same side of the $\Xi$ so that its opinion has the same sign as the $\Xi$, but the $S_{l}$ is considered to be of the reverse sign and direction to the $\Xi$. Therefore when there exists emotional contagion from the information to the public, we set the followings as prerequisites.

The strength, hence the force, on the public opinion to make move toward or away from the opinion of information is given by a function of the superimposed areas $S_{2}$ and $S_{1}$, respectively, of the two fuzzy distribution functions.

The influence the public exerted from the information, totally including the rational and emotional components, is given by a function of $S\left(\equiv S_{1}+S_{2}\right)$.

As for those functions, we adopt the power-law type functions such as $S_{2}^{\gamma}, S_{1}^{\gamma}$ and $S^{\gamma^{\prime}}$, where $\gamma$ and $\gamma^{\prime}$ are parameters.

\subsection{Node Dynamics}

According to the above model, the central opinion of a node $\xi$, varies its value depending on the force originating from the information. Hence when the information $j$ is released into the networks at $t=t_{0}$, the dynamics of the central position $\xi_{\mathrm{ki}}$ of the $i$ 'th node in the $k^{\prime}$ th network is given by

$$
\frac{d^{2} \xi_{k i}}{d t^{2}}=-\eta_{k i} \frac{d \xi_{k i}}{d t}+\varsigma_{k i}\left(S_{2}^{\gamma}-S_{1}^{\gamma}\right) \cdot \delta\left(t_{0}\right)+\mu_{k i}\left(\xi_{k i}^{0}-\xi_{k i}\right)
$$

where $\eta_{k i}, \varsigma_{k i}$ and $\mu_{k i}$ are the constants depending on the node $k i$. The first term on the right hand side is the resistant force which increases in proportion to the velocity of change $d \xi_{k i} / d t$, the second term is the force from the information and $\delta(t)$ is Dirack's delta function. The third term gives a force for the public opinion to recover its original value $\xi_{k i}^{0}$. The general solution of the above equation is given by

$$
\xi_{k i}(t)=\xi_{k i}^{0}+\frac{\varsigma_{k i}}{2 \mu_{k i}}\left(S_{2}^{\gamma}-S_{1}^{\gamma}\right) \cdot H\left(t_{0}\right) \exp \left(-\frac{\eta_{k i} \tau}{2}\right)\left\{\exp \left(\lambda_{1} \tau\right)+\exp \left(\lambda_{2} \tau\right)\right\}
$$

where $\tau \equiv t-t_{0}$, and $H(t)$ is Heaviside function such that $H(t)=1$, $1 / 2$ and 0 in the cases of $t>0, t=0$ and $t<0$, respectively. The quantities $\lambda_{1}$ and $\lambda_{2}$ are given by

$$
\lambda_{1,2}= \pm \frac{1}{2}\left(\eta_{k i}^{2}-4 \mu_{k i}\right)^{1 / 2}
$$

Hence the final form of the solution varies depending on the sign of a quantity $D \equiv \eta_{k i}{ }^{2}-4 \mu_{k i}$; either of an exponentially varying function or an oscillatory function, but both of them certainly decrease with time owing to the leading term $\exp \left(-\eta_{k i} \tau / 2\right)$.

When the public repeatedly comes to contact with the information similar to the opinion of his own, or even when he encounters the information different from his own, it generally leads the enhancement of his confidence to strengthen his belief [34], hence the deviation of his opinion from its central value becoming small with time. Also for the deviation $\sigma_{k i}$, therefore, the following dynamical equation becomes to hold.

$$
\frac{d^{2} \sigma_{k i}}{d t^{2}}=-\eta_{k i}^{\prime} \frac{d \sigma_{k i}}{d t}+\varsigma_{k i}^{\prime} S^{\gamma^{\prime}} \cdot \delta\left(t_{0}\right)+\mu_{k i}^{\prime}\left(\sigma_{k i}^{0}-\sigma_{k i}\right)
$$

where the prime represents the value for the $\sigma$, the force exerted from the information is assumed to be proportional to the $\left(\gamma^{\prime}\right)$ 'th powers of the superimposed area $S$. The solution of this equation has a similar form as Eq.(8) except that it is restricted within a limited range of $\left[\sigma_{\min }, \sigma_{\max }\right]$ as

$$
\sigma_{k i}(t)=\sigma_{k i}^{0}+\frac{\zeta_{k i}^{\prime}}{2 \mu_{k i}^{\prime}} S^{\gamma^{\prime}} \cdot H\left(t_{0}\right) \exp \left(-\frac{\eta_{k i}^{\prime} \tau}{2}\right)\left\{\exp \left(\lambda_{1}^{\prime} \tau\right)+\exp \left(\lambda_{2}^{\prime} \tau\right)\right\}
$$

where $\sigma_{k i}$ does not decrease below $\sigma_{\min }$ nor increase above $\sigma_{\max }$. By using the time varying functions $\xi_{k i}(t)$ and $\sigma_{k i}(t)$, the distribution of the opinion of the node $k i$ at a time $t$ is given by Eq.(4) by respectively replacing $X$ and $\sigma_{f}$ with $\xi_{k i}$ and $\sigma_{k i}$.

\subsection{Distribution of Collective Opinion}

When the propaganda or the political campaigns are competitively carried out, the information from different standpoints is in general released in the society at almost the same time. Under the condition that such a situation continues during a long time, we derive the intensity $z(x, t)$ for the collective opinion to be a value $x$ at a time $t$ as

1. At the first time we give a central value $\Xi$ of the information released at a time $t 0$.

2. After the calculation of $\mathrm{S} 1$ and $\mathrm{S} 2$ for every node in the k'th network, we further derive $\xi(\mathrm{t})$ and $\sigma(\mathrm{t})$ by using Eqs.(8) and (11).

3. By using $\xi(t)$ and $\sigma(t)$ thus obtained, we derive the distribution of opinion of the node at the time $t$ from Eq.(4).

4. We consider the reach $\omega \mathrm{kmi}$, that is the probability for the information to reach the node $i$ on the m'th layer.

5. We then make a statistical average of the opinion over all networks.

6. Exerting the above processes (i) (v) for the information released at different time $\mathrm{t} 0$, we make a summation of their results; namely

$$
z(x, t)=\frac{1}{N} \sum_{t_{0}} \sum_{k} \sum_{m} \sum_{i}\left\{1-\omega_{k m i}+\omega_{k m i} F_{k m i}\left(x, t: t_{0}, \xi\right)\right\} H\left(t-t_{0}\right)
$$


where $N$ is the total number of nodes, and $F_{k m i}\left(x, t: t_{0}, \xi\right)$ is the distribution of opinion at the time $t$ whose central value was $\xi$ at the initial time $t_{0}$, and $\omega_{k m i} \equiv \omega\left(t: m-1, i{ }^{\prime} \rightarrow m, i\right)$ is the probability for the information to reach or to be transmitted from the node $i$ ' on the $(m-1)$ 'th layer to the node $i$ on the next $m$ 'th layer. The larger grows the value of $\omega_{k m i}$, the deeper is the information transmitted to a layer of the network, that is to the wider range of the society. From the requisite (2) before described, we adopt the following relation for $\omega_{k m i}$.

$$
\omega\left(t: m-1, i^{\prime} \rightarrow m, i\right)=\prod_{q=1}^{m-1} S_{\mathrm{q}}^{\gamma^{\prime}}
$$

where the product on the right hand side is performed along the path (edge) toward the node from the first layer to the ( $m-1)$ 'th layer. The quantity $S_{q}$ is the superimposed area given by Eq.(6) on the $q$ 'th layer along the path.

The extent of the reach of information to an arbitrary layer $m, \Omega_{m}(t)$, is statistically given by

$$
\Omega_{m}(t)=\frac{1}{N^{\prime}} \sum_{t_{0}} \sum_{k} \sum_{q=1}^{m-1} \sum_{i^{\prime}=1}^{n_{q}} \omega\left(t: q, i^{\prime} \rightarrow q+1, i\right) H\left(t-t_{0}\right)
$$

where $N^{\prime}$ is the total number of relevant nodes, and $n_{q}$ is the total number of nodes on the $q$ 'th layer. Figure 2(d) shows the behavior of $\Omega_{m}(t)$ up tp $m=5$ under two different conditions.

\section{Numerical Calculation}

\subsection{Characters of Nodes and Information}

When we consider the set of all nodes as a whole, the central values of the opinion of nodes at an initial time, $\xi_{0}$, possibly have a sort of spectrum $\varphi\left(\xi_{0}\right)$. As for this spectrum we consider four cases such as

1. the case where all nodes have the same and unique opinion $(=\mathrm{p})$, namely $\varphi(\xi 0)=\delta(\mathrm{p})$.

2 . the case where the $\xi 0$ randomly distributes within the range $\xi_{0} \in[0,1]$.

3. the case where two opinion bumps appear centering around $\xi_{1}{ }^{0}$ and $\xi_{2}{ }^{0}$, that is

$$
\phi\left(\xi_{0}\right)=a_{1} \varphi\left(\xi_{0}: \xi_{1}^{0}, \sigma_{1}^{0}\right)+a_{2} \varphi\left(\xi_{0}: \xi_{2}^{0}, \sigma_{2}^{0}\right)
$$

where $a_{1}$ and $a_{2}$ are constants, $\varphi\left(\xi: \xi^{0}, \sigma^{0}\right)$ is the normal distribution function truncated at $\xi=0$ and 1 with a center $\xi^{0}$ and the standard deviation $\sigma^{0}$. The initial value for each node is randomly determined from the above Eq.(15).

4. the case where $\varphi\left(\xi_{0}\right)$ has an arbitrary spectrum. Also in this case the initial value is randomly determined from this spectrum.

Along with the spectrum of $\xi_{0}$, the spectrum for the central value of information, $\Xi$, is also required, about which the same types as (a) (d) are assumed. Figure 3 schematically shows the forms of $(a) \sim(d)$.
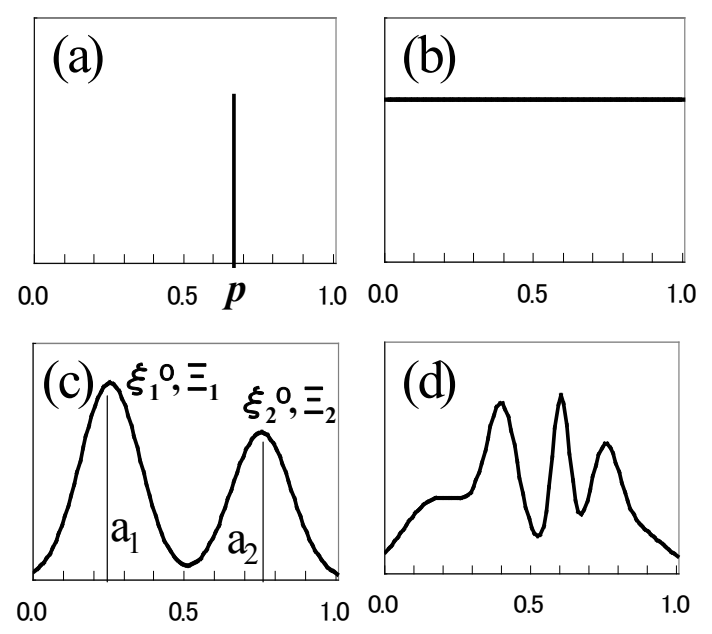

Figure 3. The conceptual forms (a) (d) for the initial distribution of $\xi_{0}$ and $\Xi$.

On the other hand, the coefficients appeared in the expressions of $\xi(t)$ and $\sigma(t)$ may probably have different values with nodes because of the assumption of each node corresponding to each individual people. There exist opinion models assuming the heterogeneity of network elements [5]. Also in our model, the heterogeneity is introduced for the character of nodes such that the set of coefficient $\eta_{k i}$ distributes in a form of normal distribution truncated at $\eta_{\min }$ and $\eta_{\max }$ with a center $\eta_{0}$ and the standard deviation $\sigma_{\eta}$. The node with a large $\eta$ is in general conservative against the environmental change so that it does not respond promptly to the transmitted information. Setting $\eta$ as a main variable in what follows, we simply assume $\varsigma=v_{1} \eta, \mu=v_{2} \eta, \eta^{\prime}=v_{0} \eta, \varsigma^{\prime}=v_{1} \eta^{\prime}$, and $\mu^{\prime}=v_{2} \eta^{\prime}$. Here $v_{0}$, $v_{1}$ and $v_{2}$ are parameters with the order of $O\left(v_{0}\right)=O\left(v_{1}\right)=O\left(v_{2}\right)=1$. In the following calculation, we set

$$
\begin{gathered}
\eta_{\min }=0.4, \eta_{\max }=1.6, \eta_{0}=1.0, \sigma_{\eta}=0.2, \sigma_{s}=0.16, \\
\sigma_{n}=0.08, \sigma_{\min }=0.04, \sigma_{\max }=0.12, \gamma=\gamma^{\prime}=1.0, \\
\text { and } v_{0}=v_{1}=v_{2}=1.0 .
\end{gathered}
$$

These values do not essentially affect our qualitative results, with which we will show some numerical examples for the characteristic behavior of collective opinion.

\subsection{Example Calculation for the Distribution of Collective Opinion}

\subsubsection{The Case for the Input of Information for Once}

We assume here that the monochromatic information, that is the one with a spectrum of Figure $3(\mathrm{a})$ with $(p=) \Xi=0.5$, is released to the first node $(m=1)$ in the 800 networks $(K=800)$ whose ramified structure differ from each other. On the other hand the homogeneous distribution as given by Figure 3(b) is assumed for the initial opinion of nodes in those networks. In the numerical calculation the central opinion $\xi$ and the deviation $\sigma$ at the time $t$ are first derived for all nodes by using Eqs.(8) and (11). Then with those quantities, the probability for each node to take an opinion $x$ is derived by using Eq.(4), and then the strength of collective opinion by Eq.(12). After 
repeatedly carrying out those processes with a finite time step $\Delta t$, the time varying distribution of collective opinion within $x \in[0,1]$ is finally derived. In what follows we set $\Delta t=1$.

Figure 4 shows the time behavior of the collective opinion thus derived, averaged up to the 3 'rd layer of all networks. The collective opinion distributes around a peak at $x=0.5$, which appears under the information with $\Xi=0.5$. The appearance of this peak is due to the gathering of the nodes on both sides of $x=0.5$ toward $x=0.5$ owing to the force from the information, and its disappearance with time to the natural return of each node to the respective original opinion. When we plot the time on the abscissa and the $\xi_{i}(t)\left(i=1 \ldots i_{\max }\right)$ on the ordinate, the resulting figure of $\xi_{i}(t)$ is such that the public opinion agglomerated at $t=0$ at around $x=0.5$ becomes to be dispersed with time into a homogeneous distribution, which is different from the traditional figures of gradual cohesion of public opinion in the real world. As seen in this figure, the intensity of collective opinion $z(x, t)$ decreases at around extreme limits $x \rightarrow 0$ and $x \rightarrow 1$. This is because the individual opinion is assumed to disperse in an form of normal distribution given by Eq.(4) so that there exists no contribution of distributional tails from $\xi<0$ and $\xi>1$ around those respective limits when we calculate the $z(x, t)$ with Eq.(12).

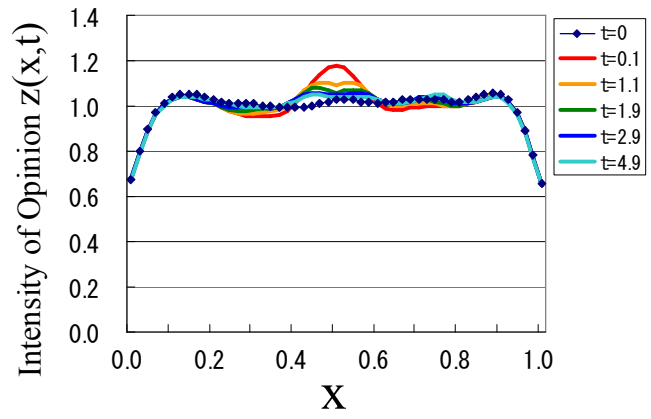

Figure 4. Time behavior of the collective opinion for the case 1 .

\subsubsection{The Case for the Repeated Input of Information}

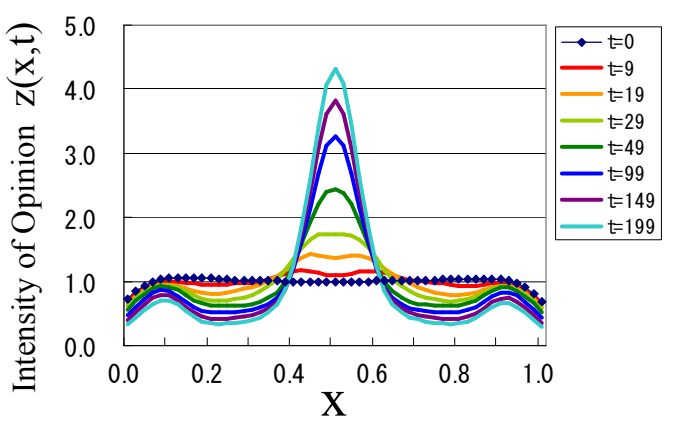

Figure 5. Time behavior of the collective opinion for the case 2 .

Figure 5 shows the behavior of collective opinion, derived by averaging over the nodes up to the 5 'th layer, when the same information as the previous case 1 is repeatedly released in the networks by 40 times with every $5 \Delta t$ period. With such repeated release of information, there appear a strong peak of collective opinion around the center and two weak peaks on the respective two wings. The latter two peaks are just apparent since the nodes near $x=0.5$ are drawn toward the center so that the density of nodes becomes decreased in between the center and the extreme limits $x=0$ and 1 to result in the remnants of nodes on both wings.

\subsubsection{The Case for the Central Opinions of the Node and the Information Different from Each Other}

Figure 6 shows the time behavior of the collective opinion obtained under the same repeated release of information as the previous case 2 but $\Xi=0.25$, which is put in the networks of the nodes with a monochromatic initial condition of $(p=)$ $\xi_{0}=0.5$; namely both the distributions are of the type (a) in Figure 3 . The collective opinion is gradually drawn toward the central opinion of information, namely toward $x=0.25$, and moreover the width of the distribution becomes narrow so that the peak of collective opinion becomes sharp with time. The behavior of the deviation $\sigma(t)$ is exemplified in Figure 7. Since the $\sigma(t)$ quickly approaches the minimum value set at first, $\sigma_{\min }$, the distribution of the opinion becomes sharp at a relatively early stage in the case of repeated receive of the information. Although even when we set the $\sigma$ constant without assuming its time variation, we can obtain the similar figure of result as Figure 6 but with the width of the peak somewhat wider than shown in that figure. Thus the behavior in Figure 6 indicates a general trend of the collective opinion. Therefore Figure 6 well represents the real situation [34] such that the public opinion sharply agglomerates when it is exerted repeated exposures of the information with a unique content. Although in the real world the agglomeration of the public opinion is owed to the interaction of the public with community members, the unification of the opinion can be attained in the cyber world by the emotional contagion, by which means the public opinion being drawn towards the opinion of information to result in the agglomeration.

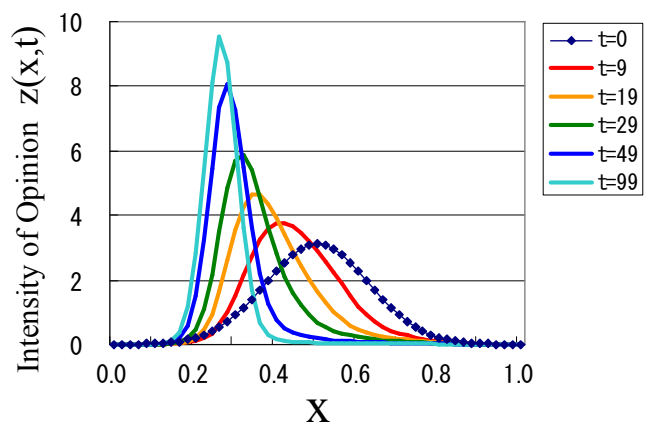

Figure 6. Time behavior of the collective opinion for the case 3.

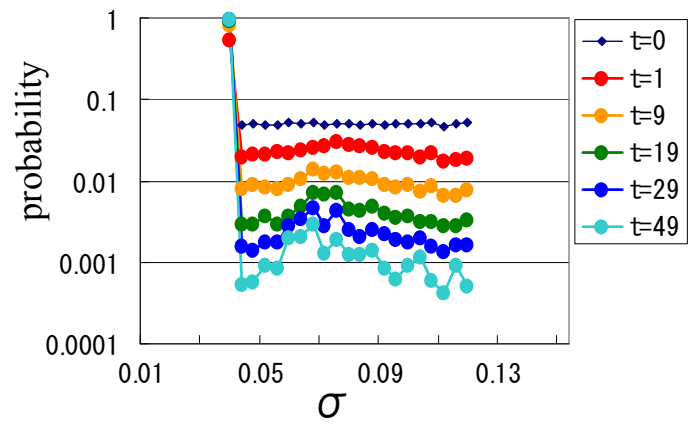

Figure 7. The probability of node of which deviation take a value $\sigma(t)$ under the same condition as Figure 6, where $\sigma_{\min }=0.04$ and $\sigma_{\max }=0.12$. 


\subsubsection{The Case for the Node Characters in the Right and Left Wings Different from Each Other}

We here consider the case where the central values, $\xi_{0}$ and $\Xi$, of both the initial public opinion and the information are of the type (c) in Figure 3 with bipolar characters of the right and left wings. As for the distribution of the nodes we set here $\xi_{1}^{0}=0.25$, $\xi_{2}{ }^{0}=0.75, a_{1}=a_{2}=0.5, \sigma_{1}{ }^{0}=\sigma_{2}{ }^{0}=0.15$, and for the information $\Xi_{1}=0.25, \Xi_{2}=0.75, a_{1}=a_{2}=3.0 、 \sigma_{1}=\sigma_{2}=0.1$. So far as the node character is symmetrical with respect to $x=0.5$, the distribution of the collective opinion becomes symmetric with $x=0.5$ even if the spectrum of released information is asymmetric with $x=0.5$. When the node character is asymmetric with $x=0.5$, however, it appears the asymmetric peculiarity in the final distribution of collective opinion. Figure 8 shows such an example for the case of different value of $\eta$ on the right and left wings such as $\eta(x>0.5) / \eta(x \leq 0.5)=3.0$.

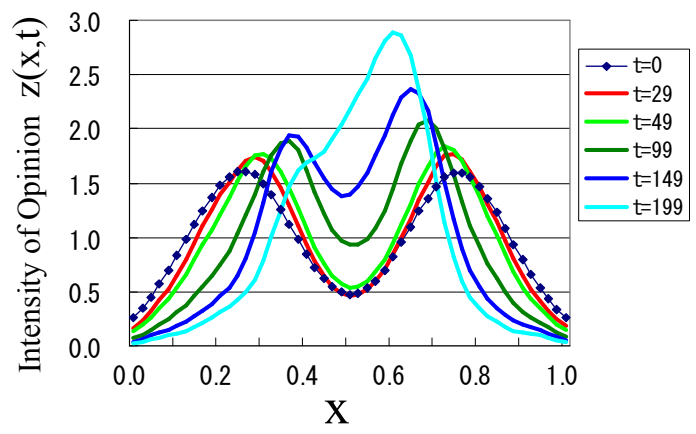

Figure 8. Time behavior of the collective opinion for the case 4.

In our model the quantity $\eta$ represents the inertia against the change of individual's opinion, as described before, and is related to the time scale for the opinion to naturally recover its original value as seen in Eq.(8). When the information spectrum has a bipolar character and the nodes are repeatedly exposed to such information, the distributions of the collective opinion on both wings gradually become sharp (because of the decrease of $\sigma$ ) and the wing in the region $x \geqq 0.5$ gradually grows large in its area compared to the wing in the region $x<0.5$. Such a behavior of the distribution is a result of competition between the recovery of the original opinion of node and the opinion change brought by the exposure of information, that is, the competition between the time scale in terms of $\eta$ and the frequency of the exposure. When the repeated exposure of information further continues, the collective opinion becomes to broadly gather around the center $x=0.5$. This is because the nodes on either side of right and left wings are drawn toward the central direction due to the influence from both sides of wings of information. Hence the final form of collective opinion broadly but asymmetrically distributes around the center.

In what follows we study the collective opinion by providing more realistic data.

\section{Application to the Case of 2016 USA President Election}

During the period of 2016 USA President election, many sorts of fake news and public relations are flooded in the cyber world so that it has been widely discussed on what extent the social media has influenced on the attitude of the public regarding the election [34-38]. Here we estimate, by using our model and the data at the time of the election, on what extent the result of election was changed if the public is assumed to have reacted to the information released through the SNS. We assume in the followings that the information sent from a server to the node on the first layer $(m=1)$ is totally received to that first node (that is, the extent of the reach $\omega$ is 1.0), and therefore the following simulation is for the subgroup of the public who usually uses SNS in everyday life.

As shown in Figure 6, the peak of the collective opinion changes its position depending on the spectrum of information. When the period of the release of information is shorter than the time scale for the node to recover its original position, the opinion peak of the node gradually moves toward the opinion of the maximum strength of information to result in the change of the distribution of collective opinion. When the time scale above cited differs in the right and left wings, the fraction of the public belong to each wing gradually changes from the initial value since the effect on each wing differs from each other. Here we measure the effect as the fraction of the public that moves to the opposite side of wing across $x=0.5$.

Figure 9(a) shows the spectrum of information released in the SNS (Twitter) at the time of the election [39]. In this figure the relative frequency for the release of information is given for the seven political positions with the same bin from the extreme bias (left) to the extreme right (bias) according to [39], assuming that the extreme limits of the extreme left and extreme right corresponding to $x=0$ and $x=1$, respectively. The amount of information released in the left wing $(x<0.5)$ should be noticed to overwhelm the one in the right wing.
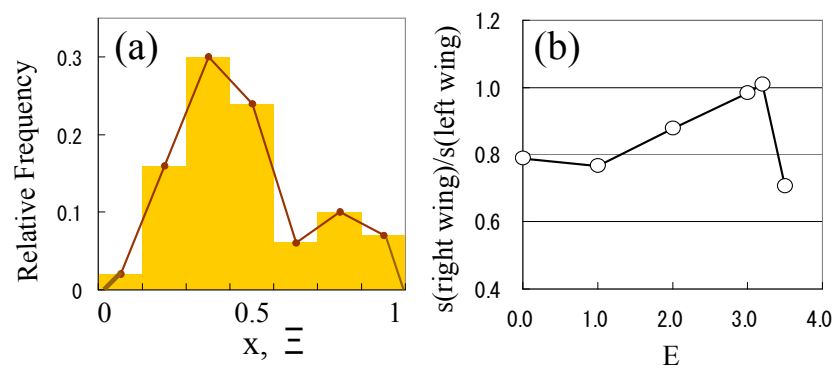

Figure 9. (a) Spectrum of the opinion of information released at the time of the election [39] shown with bars and the continuous spectrum used in our simulation with lines, which is the type (d) in Figure 3. (b) E-dependency of the ratio $s$ (right wing)/s(left wing).

The initial number ratio of the public belonging to the right wing with $x \in[0.5,1.0]$ to that to the left wing with $x \in[0.0,0.5)$ is assumed as 0.8 (namely $\mathrm{a}_{1}=1.0, a_{2}=0.8$ in Eq.(15)). Moreover the central value of the initial opinion of node, $\xi_{0}$, is also assumed to normally distribute around $x=0.2$ and 0.8 in the left and right wings, respectively, with both a common deviation $\sigma^{0}=0.15$, such as the form (c) in Figure 3 . 
At the time of 2016 election, it is reported [40] that the information was released through the SNS by about $3.7 \times 10^{7}$ times and shared by a total of $7.6 \times 10^{8}$ users, and that the websites consisted of fake news were accessed by $1.59 \times 10^{8}$ times during the period of election. Although the precise number of the exposure of information per person is unknown, we simply assume in what follows that the information by the social media with a spectrum given by Figure 9(a) is released by 40 times to the first node of each network. The repeated release of information as such during a short period affects not only the final quantity but also the quality of collective opinion. The quantity $\eta$ is variable in our model between the left and right wings, so that by using $\eta$ we introduce anew a parameter $E$ defined by $E \equiv \eta$ (right wing) $/ \eta$ (left wing). This quantity $E$ is the ratio of the extent for the public in the right and left wings to lose interest in the information, since the $\eta$ is related to the recovering time scale of opinion. It is pointed out that there exists clear difference in the political characters of the public between the two wings [38] so that the condition $E \neq 1$ is highly probable.

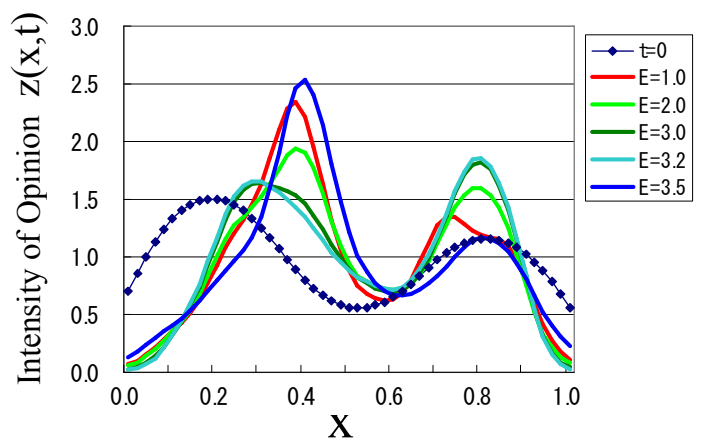

Figure 10. Change of the distribution of collective opinion with the parameter E at $t=199$.

Figure 10 shows the behavior of the collective opinion with the variation of $E(\leq 3.25)$ at the time $\mathrm{t}=199$, where the exposure period of information is $5 \Delta t=5$. Although the central position of the collective opinion peak in $x \in[0.5,1.0],<x$ (right wing) $>$, is almost constant irrespective to the variation of $E$, the peak in $x \in[0,0.5)$ has a tendency to move toward the center, $x=0.5$. Such an asymmetric behavior originates from the asymmetries of the spectrum of input information and of the political character of the public on both sides of opinion wings.

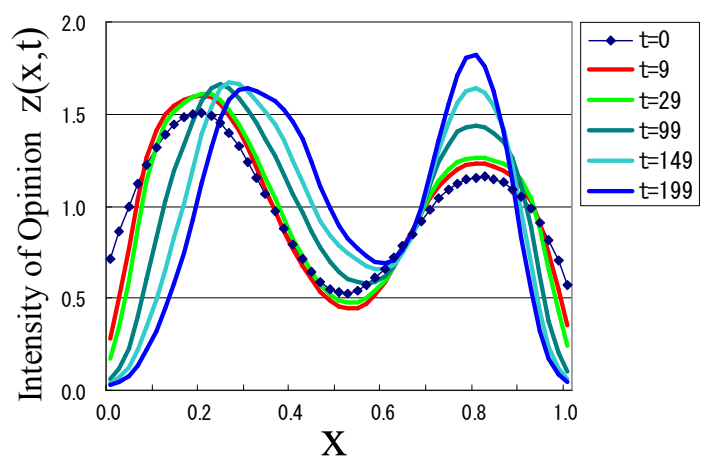

Figure 11. Time variation of the collective opinion for the case of $E=3.25$.
Figure 11 shows the time variation of the collective opinion with $E=3.25$. when the time, hence the number of the exposure of information is treated as a parameter. Here the same period $5 \Delta t(=5)$ as in Figure 10 is assumed for the exposure. In this case with increasing the number of the exposure, the peak in the right wing becomes sharp and its maximum value $a($ right $)$ grows large. On the other hand, although the width of the left-wing peak is almost invariable, its position gradually moves toward the center $x=0.5$.

We now represent the ratio of the areas in the regions of $x \in[0.0 .0 .5)$ and $[0.5,1.0]$ below the curve after 40 times release of information (that is the curve at $\mathrm{t}=199$ in Figure 11), $\mathrm{s}($ right wing)/s(left wing) (or simply $s(r) / s(l))$, as the ratio of the number of supporters for politically left and right wings. The ratio $s(r) / s(l)$ changes with the variable $E$ as given in Figure (b). We should note here that the ratio, its initial value being 0.8 at $E=1$, increases with $E$ to become almost unity or slightly over unity, indicating a balance or a reversal at $E \approx 3.2$ between the right and left wings. Such a movement of supporters from the left to the right gradually increase with the number of the exposure of information, and the collective opinion becomes to change its form to a broad distribution centering around $x=0.5$, such trends being well expected from Figure 8. If we adopt the result at $t=199$ in Figure 11 as a final distribution, we can see there two clear peaks of supporters each of which is in the right and left respective wings, just as the case of 2016 President election. We point out, therefore, that the reversal of the number of supporters between the right and left wings is a quite possible matter. Moreover the occurrence of such a reversal, according to Figure 11, is due to the move of people in the left of center to the right of center, without the participation of the core of the left wing in such a reversal. Hence we further point out that one of the causes of the unexpected turn in the election is possibly due to the mechanism as such in the cyber world.

\section{Conclusion}

Public cognition and feeling on a certain matter vary from time to time under the influence of surrounding environment without holding definite and discrete values. They fluctuate around central values so that they are fuzzy in nature. On the other hand, the information released in the cyber world has its individual stance that distributes within a certain range of value. Hence it is also fuzzy in the meaning of semantics. When the public interacts with the information as such, the resultant effect becomes also to be fuzzy in the double sense since the interpretation of information depends on the subjectivity of the receiver. In this paper we have proposed a methodology to treat the collective opinion formed by the public with the fuzzy distribution of individual opinion, which is exposed by the information from the social media with the fuzzy distribution of value.

The public attitude or opinion to a certain matter have been treated in physics as discrete values by assuming that their change is a result of the direct contact of individual people with the neighboring public or with the news media. Although 
such an approach is appropriate for the phenomena in the real world, different approaches may be required for treating the change of opinion in the cyber world where the emotional contagion seems to be significant in interacting with information. In this paper by introducing a concept of fuzzy theory with fuzzy functions of the form of normal distribution, we have statistically modeled the collective opinion which is realized in the cyber world as a collective result of the interaction of individual people with the information released in the cyber network.

According to the numerical calculation, it became clear that, also in the cyber world where the public do not interact with each other, the public opinion is drawn by the opinion of social media finally to gather toward a certain value of opinion. It also became clear the easy occurrence of the so-called echo-chamber effect. By applying our method to the case of 2016 USA President election, it was found a possibility of the reversals of the number of supporters in the right and left political wings in the case of the different characters of supporters between the right and left side wings. With the similar approach to this, we may be able to study the influence of the social media on the collective opinion regarding some matters such as the global warming $[40,41]$ and the new type corona virus [42].

Although the SNS becomes to be a mean, at present, to widely transmit information, the rate of the public is not necessarily large, who usually use only the SNS for obtaining the everyday information without receiving any information from the media in the real world, Since the opinion of an individual people to a certain matter is represented as a sum of contributions from both real and cyber worlds, the change of the opinion only in the cyber world may not be so important as a whole. Notwithstanding, our method is a useful approach to estimate the extent of influence of the social media on the public so that we expect to improve our model by accumulating more realistic data $[43,44]$ and analyzing by using those data [26], especially the data of the time scale for the public to forget the information, the time scale [45] for the information to spread in the network, and the frequency and the spectrum for the information to be released in the networks.

\section{References}

[1] C. Castellano, S. Fortunato, V. Loreto, Statistical physics of social dynamics, Rev. Mod. Phys. 81 (2009) 591-646.

[2] L. Neuhauser, A. Mellor, R. Lambiotte, Multibody interactions and nonlinear consensus dynamics on network systems, Phys. Rev. E 101 (2020) 032310, and references therein.

[3] X. Dong, Y. Liu, C. Wu, Y. Lian, D. Tang, A double-identity rumor spreading model, Physica A 528 (2019) 121479.

[4] M. NeKoree, Y. Moreno, G. Bianconi, M. Marsili, Theory of rumor spreading in complex social network, Physica A 374 (2007) 457-470.
[5] J. Ma, H. Zhu, Rumor diffusion in heterogeneous networks by considering the individual subjective judgment and diverse characteristics, Physica A 499 (2018) 276-287, and references therein.

[6] T. Ohnishi, Can interactive dual field of information explain the prevalent phenomena, Am. J. Phys. Appl. 7 (2019) 144-155.

[7] Y. Lan, Z. Lian, R. Zeng, D. Zhu, Y. Xia, M. Liu, P. Zhang, A statistical model of the impact of online rumors on the information quantity of online public opinion, Physica A 541 (2020) 123623.

[8] Y. Yi, Z. Zhang, C. Gan, The outbreak threshold of information diffusion over social-physical networks, Physica A 526 (2019) 121128.

[9] M. Del Vicario, A. Scala, G. Caldarelli, H. E. Stanley, W. Quattrociocchi, Modeling confirmation bias and polarization, Sci. Rep. 7 (2017) 40391.

[10] H-W. Lee, N. Malik, F. Shi, P. Mucha, Social clustering in epidemic spread on coevolving networks, Phys. Rev. E 99 (2019) 062301.

[11] P. Jia, C. Wang, G.. Zhang, J. Ma, A rumor spreading model based on two propagation channels in social networks, Physica A 524 (2019) 342-353.

[12] Y. Lian, X. Dong, Y. Liu, Topological evolution of the internet public opinion, Physica A 486 (2017) 567-578.

[13] X. Yin, H. Wang, P. Yin, H. Zhu, Agent-based opinion formation modeling in social network: a perspective of social psychology, Physica A 532 (2019) 121786.

[14] N. Askitas, Explaining opinion polarization with opinion copulas, PLOS ONE 12 (2017) e0183277.

[15] R. Zeng, D. Zhu, A model and simulation of the emotional contagion of netizens in the process of rumor reputation, Sci. Rep. 9 (2019) 14164.

[16] A. D. I. Kramer, J. E. Guillory, J. T. Hancock, Experimental evidence of massive scale emotional contagion through social networks, PNAS 111 (2014) 8788-8790.

[17] A. Chmiel, J. Slenkiewicz, M. Thelwall, G. Paltoglou, K. Buckley, A. Kappas, J. A. Holyst, Collective emotions online and their influence on community life, PLOS ONE 6 (2011) e22207.

[18] R, Fan, K. Xu, J. Zhao, A agent-based model for emotion contagion and competition in online social media, Physica A 495 (2018) 245-259.

[19] A. L. Hill, D. G. Rand, M. A. Nowak, N. A. Christakis, Emotions as infections diseases in a large social networks; the SISa model, Proc. Roy. Soc. B 277 (2010) 3827-3835.

[20] E. Ferrara, Z. Yang, Measuring emotional contagion in social media, PLOS ONE (Nov. 6, 2015) e0142390.

[21] E. Cox, The fuzzy systems handbook: a practitioner's guide to building, using, maintaining fuzzy systems (AP Professional, Boston, 1994).

[22] M. Kochen, Applications of Fuzzy Sets in Psychology (Academic Press, 1974).

[23] A-L. Barabasi, R. Albert, Emergence of scaling in random network, Science 286 (1999) 509-512. 
[24] A-L. barabasi, R. Albert, H. Jeong, Mean field theory for scale-free random network, Physica A 272 (1999) 173-187.

[25] D. J. Watts, A simple model of global cascades on random networks, PNAS 99 (2002) 5766-5771.

[26] A. Bessi, F. Zollo, M. Del Vicaro, M. Puliga, A. Scala, G. Caldarelli, B. Uzzi, W. Quattrociocchi, Users polarization on Facebook and Youtube, PLOS ONE 11 (2016) e0159641.

[27] A. Boutyline, R. Willer, The social structure of political echo chambers: variation in ideological homophily in online networks, Pol. Psycho. 38 (2017) 551-569.

[28] N. Grinberg, K. Joseph, L. Friedland, B. Swire-Thompson, D. Lazer, Fake news on Twitter during the 2016 U. S. Presidential election, Science 363 (2019) 374-378.

[29] A. Bovet, F. Marone, H. A. Makse, Validation of Twitter opinion trends with national polling aggregates: Hillary Clinton vs Donald Trump, Sci. Rep. 8 (2018) 8673.

[30] T. Lee, The global rise of "fake news" and the threat to democratic election in the USA, Pub. Admini. Policy, 22, (2019) 15-24.

[31] B. Monsted, P. Sapiezynski, E. Ferrara, S. Lehmann, Evidence of complex contagion of information in social media: an experiment using Twitter bots, PLOS ONE 12 (2017) $\mathrm{e} 01841482$.

[32] M. Maciel, A. C. R. Martins, Ideologically motivated biases in a multiple issue opinion model, Physica A 553 (2020) 124293.

[33] L. Zhao, J. Wang, R. Huang, H. Cui, X. Qiu, X. Wang, Sentiment contagion in complex network, Physica A 394 (2014) 17-23.

[34] C. A. Bail, L. P. Argyle, T. W. Brown, J. P. Bumpus, H. Chen, M. B. Fallin Hunzaker, J. Lee, M. Mann, F. Merhout, A. Volfovsky, Exposure to opposing views on social media can increase political polarization, PNAS 115 (2018) 9216-9221.
[35] C. A. Bail, B. Guay, E. Maloney, A. Combs, D. Sunshine, D. S Hillygus, F. Merhout, D. Freelon, A. Volfovsky, Assessing the Russian Internet Research Agency's impact on the political attitudes and behaviors of American Twitter Users in late 2017, PNAS 117 (2020) 243-250.

[36] D. R. Dewhurst, C. M. Danforth, P. S. Dodds, Noncooperative dynamics in election interference, Phys. Rev. E 101 (2020) 022307.

[37] E. Ferrara, O. Varol, C. Davis, F. Menczer, A. Flammini, The rise of social bots, Com. ACM 59 (2016) 96-104.

[38] H. Allcott, M. Gentzkow, Social media and fake news in the 2016 election, J. Economic Perspectives 31 (2017) 211-236.

[39] A. Bovet, H. A. Makse, Influence of fake news in Twitter during the 2016 US presidential election, Nature com. 10 (2019) 1-14.

[40] for instance, BBC News, What does Trump actually believe on climate change https://www.bbc.com/news/world-us-canada-51213003 (retrieved Sept 9, 2020).

[41] J. S. Damico, M. Baildon, A. Panos, Media literacy and climate change in a post-truth society, J. Media Literacy Edu. 10 (2018) 11-32.

[42] N. Fleming, Fighting coronavirus misinformation, Nature 583 (2020) 155-156.

[43] D. Mocanu, L. Rossi, Q. Zhang, M. Karsai, W. Quattrociocchi, Collective attention in the age of (mis) information, Comp. Human Behav. 51 (2015) 1198-1204.

[44] M. Del Vicario, A. Bessi, F. Zollo, F. Petroni, A. Scala, G. Caldarella, H. E. Stanley, W. Quattrociocchi, The spreading of misinformation online, PNAS 113 (2016) 554-559.

[45] S. Vosoughi, D. Roy, S. Aral, Science 359 (2018) 1146-1151. 